\title{
Neuropsychiatric symptoms predict hypometabolism in preclinical Alzheimer disease OPEN
}

Kok Pin Ng, MRCP

Tharick A. Pascoal, MD

Sulantha

Mathotaarachchi, MSc Chang-Oh Chung, MD

Andréa L. Benedet, MSc Monica Shin, MSc

Min Su Kang, BSc

Xiaofeng Li, MD

Maowen $\mathrm{Ba}, \mathrm{MD}$

Nagaendran Kandiah, FRCP

Pedro Rosa-Neto, MD, $\mathrm{PhD}$

Serge Gauthier, MD For the Alzheimer's

Disease Neuroimaging Initiative

Correspondence to

Dr. Gauthier:

serge.gauthier@mcgill.ca

Supplemental data at Neurology.org

\section{ABSTRACT}

Objective: To identify regional brain metabolic dysfunctions associated with neuropsychiatric symptoms (NPS) in preclinical Alzheimer disease (AD).

Methods: We stratified 115 cognitively normal individuals into preclinical AD (both amyloid and tau pathologies present), asymptomatic at risk for AD (either amyloid or tau pathology present), or healthy controls (no amyloid or tau pathology present) using [18F]florbetapir PET and CSF phosphorylated tau biomarkers. Regression and voxel-based regression models evaluated the relationships between baseline NPS measured by the Neuropsychiatric Inventory (NPI) and baseline and 2-year change in metabolism measured by [18F]fluorodeoxyglucose (FDG) PET.

Results: Individuals with preclinical AD with higher NPI scores had higher [18F]FDG uptake in the posterior cingulate cortex (PCC), ventromedial prefrontal cortex, and right anterior insula at baseline. High NPI scores predicted subsequent hypometabolism in the PCC over 2 years only in individuals with preclinical AD. Sleep/nighttime behavior disorders and irritability and lability were the components of the NPI that drove this metabolic dysfunction.

Conclusions: The magnitude of NPS in preclinical cases, driven by sleep behavior and irritability domains, is linked to transitory metabolic dysfunctions within limbic networks vulnerable to the AD process and predicts subsequent PCC hypometabolism. These findings support an emerging conceptual framework in which NPS constitute an early clinical manifestation of AD pathophysiology. Neurology ${ }^{\circledR}$ 2017;88:1814-1821

\section{GLOSSARY}

AD = Alzheimer disease; ADNI = Alzheimer's Disease Neuroimaging Initiative; ADNI-mem = Alzheimer's Disease Neuroimaging Initiative memory composite score; $\mathbf{A l}=$ anterior insula; $\mathbf{A R - A D}=$ asymptomatic at risk for Alzheimer disease; $\mathbf{C D R}=$ Clinical Dementia Rating; [ $\left.{ }^{18} \mathrm{~F}\right] \mathrm{FDG}=\left[{ }^{18} \mathrm{~F}\right]$ fluorodeoxyglucose; $\mathbf{M C l}=$ mild cognitive impairment; $\mathbf{N P I}=\mathbf{N e u r o p s y c h i a t r i c}$ Inventory; NPS = neuropsychiatric symptoms; $\mathbf{P C C}=$ posterior cingulate cortex; $\mathbf{p}$-tau $=$ phosphorylated tau; $\mathbf{S N}=\mathbf{s a l i e n c e}$ network; SUVR = standardized uptake value ratio; vmPFC = ventromedial prefrontal cortex.

Neuropsychiatric symptoms (NPS) represent a common feature of mild cognitive impairment (MCI) and dementia phases of Alzheimer disease (AD). ${ }^{1}$ In these patients, NPS are associated with a poorer outcome in cognition and functional state. ${ }^{2,3}$ Although there is an emerging conceptual framework supporting NPS as noncognitive symptoms of preclinical AD, ${ }^{4}$ the role of NPS as early indicators of AD pathophysiologic progression remains unclear. Studies conducted in patients with $\mathrm{AD}$ indicate that NPS are associated with metabolic dysfunction in brain networks subserving mood and cognition. ${ }^{5}$ Therefore, further studies focusing on the association between NPS and AD pathophysiologic abnormalities are of paramount importance given

From the Translational Neuroimaging Laboratory (K.P.N., T.A.P., S.M., C.-O.C., A.L.B., M.S., M.S.K., P.R.-N.) and Alzheimer's Disease Research Unit (K.P.N., X.L., M.B., P.R.-N., S.G.), McGill University Research Centre for Studies in Aging, Montreal, Quebec, Canada;

Department of Neurology (K.P.N., N.K.), National Neuroscience Institute, Singapore; Montreal Neurological Institute (P.R.-N.); Department of Neurology and Neurosurgery (P.R.-N.), McGill University, Montreal, Quebec, Canada; Department of Neurology (X.L.), The Second Affiliated Hospital of Chongqing Medical University, Chongqing; and Department of Neurology (M.B.), Yantai Yuhuangding Hospital Affiliated to Qingdao Medical University, Shandong, PR China.

Data used in preparation of this article were obtained from the Alzheimer's Disease Neuroimaging Initiative (ADNI) database. The ADNI investigators contributed to the design and implementation of ADNI and/or provided data. The ADNI list is available at Neurology.org.

Go to Neurology.org for full disclosures. Funding information and disclosures deemed relevant by the authors, if any, are provided at the end of the article. The Article Processing Charge was funded by the authors.

This is an open access article distributed under the terms of the Creative Commons Attribution-NonCommercial-NoDerivatives License 4.0 (CC BY-NC-ND), which permits downloading and sharing the work provided it is properly cited. The work cannot be changed in any way or used commercially without permission from the journal. 
a growing body of evidences proposing subtle NPS as manifestations of emergent disease progression. ${ }^{6}$

$\left[{ }^{18} \mathrm{~F}\right]$ fluorodeoxyglucose $\left(\left[{ }^{18} \mathrm{~F}\right] \mathrm{FDG}\right) \mathrm{PET}$ is a technique that is sensitive in detecting changes in resting metabolism associated with the neuropsychiatric conditions ${ }^{7}$ and metabolic declines commonly observed in neurodegenerative conditions. ${ }^{8}$ In $\mathrm{AD}$, hypometabolism has been considered a part of disease pathophysiology and interpreted as synaptic abnormality or neuronal injury. Thus, modeling the magnitude of symptoms as a function of changes in brain metabolism constitutes a valuable strategy to investigate the neural correlates of NPS in preclinical AD.

Here, in a longitudinal observation of cognitively normal individuals stratified by hallmark $\mathrm{AD}$ biomarkers to identify individuals with preclinical $\mathrm{AD}$ with the highest risk of progression to clinical $\mathrm{AD},{ }^{9}$ we test the hypothesis that NPS are associated with metabolic abnormalities in limbic regions and predict pathophysiologic progression in individuals with preclinical $A D$.

METHODS Data used in the preparation of this article were obtained from the Alzheimer's Disease Neuroimaging Initiative (ADNI) database (adni.loni.usc.edu). The ADNI was launched in 2003 as a public-private partnership led by principal investigator Michael W. Weiner, MD. The primary goal of ADNI has been to test whether serial MRI, PET, other biological markers, and clinical and neuropsychological assessment can be combined to measure the progression of $\mathrm{MCI}$ and early $\mathrm{AD}$.

In this study, we selected cognitively normal individuals with baseline Neuropsychiatric Inventory (NPI) and Clinical Dementia Rating (CDR) scores, CSF phosphorylated tau (ptau $181 \mathrm{p})$ measurements, $\left[{ }^{18} \mathrm{~F}\right]$ florbetapir PET imaging, both baseline and 2-year follow-up $\left[{ }^{18} \mathrm{~F}\right] \mathrm{FDG}$ PET imaging, and ADNI memory composite score (ADNI-mem). We defined cognitively normal individuals as those with a Mini-Mental State Examination score of $\geq 24$, CDR score of 0 , and absence of any neuropsychiatric diseases such as depression, MCI, and dementia. The participants were then stratified on the basis of the recent definition of preclinical $\mathrm{AD}^{9}$ into 3 groups: healthy controls, no amyloid or tau pathology present; asymptomatic at risk for $\mathrm{AD}(\mathrm{AR}-\mathrm{AD})$, either amyloid or tau pathology present; or preclinical $\mathrm{AD}$, both amyloid and tau pathologies present. With this definition, ${ }^{9}$ the risk of progression to clinical $\mathrm{AD}$ is particularly high in preclinical AD. In AR-AD, which may represent cognitively normal individuals who do not follow the temporal sequence in the amyloid cascade hypothesis, ${ }^{10}$ the risk of clinical evolution still needs to be determined. The inclusion/exclusion criteria adopted by ADNI can be found at www.adni-info.org (accessed October 2016).

Standard protocol approvals, registrations, and patient consents. The ADNI study was approved by the Institutional Review boards of all of the participating institutions. Informed written consent was obtained from all participants at each site.
Neuropsychological assessments. The neuropsychological assessments were performed by certified raters using standardized ADNI protocols. The CDR, NPI, and ADNI-mem datasets used in this study were obtained from the ADNI files CDR.csv, NPI.csv, and UWNPSYCHSUM_01_28_15-5.csv, respectively. In this study, the NPI was used to measure NPS of the participants. The NPI is an instrument that assesses behavioral disturbances occurring in patients with dementia. ${ }^{11}$ Both the severity and frequency of each symptom are measured, and this information is obtained from a caregiver familiar with the individual's behavior. ADNI-mem is a validated composite memory score derived from data from the ADNI neuropsychological battery. ${ }^{12}$ Briefly, a modern psychometric approach was used to analyze the Rey Auditory Verbal Learning Test, AD Assessment Scale-cognition, MiniMental State Examination, and Logical Memory tests to obtain a composite memory score. In the ADNI-mem test, lower scores reflect poorer performance. Details of the ADNI protocols for the neuropsychological assessments and the methods for developing the ADNI-mem can be found at www.adniinfo.org (accessed October 2016).

CSF analysis. CSF p-tau ${ }_{181 \mathrm{p}}$ was measured with the Luminex multiplex platform (Luminex, Austin, TX) and Innogenetics INNO-BIA AlzBio3 (Innogenetics, Ghent, Belgium) immunoassay reagents. The CSF biomarker datasets used in this study were obtained from the ADNI file UPENNBIOMK5-8. csv. An ADNI published cutoff of CSF p-tau 181p $_{\mathrm{p}}>23 \mathrm{pg} / \mathrm{mL}$ was used to define the presence of tau pathology. ${ }^{13}$ Details of the ADNI methods for the acquisition and measurement of CSF can be found at www.adni-info.org (accessed October 2016).

MRI and PET methods. MRI and PET standard acquisition protocols were described in the ADNI website at http://adni. loni.usc.edu/methods/ (accessed October 2016). T1-weighted MRI images corrected for field distortions were processed with the CIVET image processing pipeline, ${ }^{14}$ and the PET images were processed with an established image processing pipeline. ${ }^{15}$ In summary, the preprocessed images from the ADNI database were spatially normalized to the Montreal Neurological Institute 152 standardized space with the use of transformations obtained for PET native to MRI native space and the MRI native to the Montreal Neurological Institute 152 space. Subsequently, the $\left[{ }^{18} \mathrm{~F}\right]$ florbetapir PET standardized uptake value ratio (SUVR) and the $\left[{ }^{18} \mathrm{~F}\right]$ FDG PET SUVR maps were generated with the cerebellum gray matter and the pons, respectively, as reference regions. $\left[{ }^{18} \mathrm{~F}\right]$ florbetapir PET images were then normalized for the white matter SUVR. The global brain glucose uptake and the global amyloid deposition were defined as the average SUVR calculated from several brain regions characteristic to $\mathrm{AD}$, including the precuneus, prefrontal, orbitofrontal, parietal, temporal, anterior, and posterior cingulate cortices in the $\left[{ }^{18} \mathrm{~F}\right] \mathrm{FDG}$ and $\left[{ }^{18} \mathrm{~F}\right]$ florbetapir PET images, respectively. Because we do not have access to the pathology data, we used the best operational point of the receiver operating characteristic curve contrasting controls and ADNI patients with $\mathrm{AD}(\mathrm{n}=90)^{16}$ and calculated $\left[{ }^{18} \mathrm{~F}\right]$ florbetapir PET SUVR $>1.15$ as the threshold for positive amyloid pathology. With this threshold, fewer than one-third of the controls were amyloid positive, which is consistent with the literature. ${ }^{17}$

Statistical methods. Statistical analyses were performed with the R Statistical Software Package version 3.3.018 with the RMINC library. Descriptive statistics and frequency distributions 
of baseline demographics and cognitive scores were summarized and compared between the 3 groups using analysis of variance for continuous measurements and $\chi^{2}$ tests for categorical measurements. Linear regression models evaluated the associations of NPI scores with baseline global $\left[{ }^{18} \mathrm{~F}\right] \mathrm{FDG}$ SUVR and global $\Delta\left[{ }^{18} \mathrm{~F}\right]$ FDG SUVR over 2 years in each group. Percentage change in $\left[{ }^{18} \mathrm{~F}\right] \mathrm{FDG}$ SUVR was defined as $\left\{\left(\Delta\left[{ }^{18} \mathrm{~F}\right] \mathrm{FDG}=\left[{ }^{18} \mathrm{~F}\right] \mathrm{FDG}\right.\right.$ baseline $-\left[{ }^{18} \mathrm{~F}\right] \mathrm{FDG}$ follow-up $) /\left[{ }^{18} \mathrm{~F}\right] \mathrm{FDG}$ baseline $\}$. An interaction term was added to the regression models to evaluate the interactions of NPI and the biomarker groups on $\Delta\left[{ }^{18} \mathrm{~F}\right] \mathrm{FDG}$ SUVR:

$$
\begin{aligned}
\Delta\left[{ }^{18} \mathrm{~F}\right] \mathrm{FDG} \text { SUVR }= & \beta_{0}+\beta_{1}(\text { baseline NPI }) \\
& +\beta_{2}(\text { biomarker groups }) \\
& +\beta_{3}(\text { baseline NPI } \times \text { biomarker groups }) \\
& + \text { covariates }+\varepsilon
\end{aligned}
$$

Linear regression models also evaluated the associations of individual subcomponents of the NPI with $\Delta\left[{ }^{18} \mathrm{~F}\right] \mathrm{FDG}$. In a secondary analysis, we used linear regression models to evaluate the associations between NPI scores and ADNI-mem at baseline and $\triangle \mathrm{ADNI}$-mem over 2 years in each group as follows: $[\mathrm{ADNI}-$ mem $=($ ADNI-mem baseline - ADNI-mem follow-up $) /$ ADNI-mem baseline].

Voxel-based regression models were used to test the associations of NPI scores with baseline $\left[{ }^{18} \mathrm{~F}\right] \mathrm{FDG}$ SUVR and $\Delta\left[{ }^{18} \mathrm{~F}\right]$ FDG SUVR over 2 years in each group:

$$
\text { Baseline } \begin{aligned}
\left.{ }^{18} \mathrm{~F}\right] \mathrm{FDG} \text { SUVR }= & \beta_{0}+\beta_{1}(\text { baseline NPI }) \\
& + \text { covariates }+\varepsilon
\end{aligned}
$$

$\Delta\left[{ }^{18} \mathrm{~F}\right] \mathrm{FDG}$ SUVR $=\beta_{0}+\beta_{1}($ baseline NPI $)+$ covariates $+\varepsilon$

We extracted and $z$ scored the mean SUVR at baseline and at follow-up from the regions where significant associations between NPI scores and $\Delta\left[{ }^{18} \mathrm{~F}\right] \mathrm{FDG}$ were observed. We defined the presence of hypometabolism if the mean $\left[{ }^{18} \mathrm{~F}\right] \mathrm{FDG}$ SUVR was significantly lower ( $95 \%$ confidence interval) than the biomarkernegative individuals, under the assumption that these individuals had a normal metabolic trajectory.

Bonferroni correction was used to correct the aforementioned linear regression analysis for multiple-comparison tests. The statistical parametric maps presented in this study were false discovery rate corrected for multiple comparisons with a threshold of $p<0.001$. All statistical models presented here were corrected for age, sex, education, and $A P O E \& 4$ status.

RESULTS Baseline demographics, $A P O E$ status, and biomarker characteristics of the 3 groups are summarized in the table.

We found that individuals with preclinical $\mathrm{AD}$ with higher NPI scores had higher $\left[{ }^{18} \mathrm{~F}\right] \mathrm{FDG}$ uptake in the posterior cingulate cortex (PCC), ventromedial prefrontal cortex (vmPFC), and right anterior insula (AI) at baseline (figure 1). However, this finding was not observed in the AR-AD or healthy control group at baseline. NPI scores did not have an effect on baseline global $\left[{ }^{18} \mathrm{~F}\right] \mathrm{FDG}$ uptake in any of the groups.

We further found that high NPI scores predicted global $\left[{ }^{18} \mathrm{~F}\right]$ FDG uptake decline over 2 years in the preclinical $\mathrm{AD}$ group $(\beta=0.52, \mathrm{SE}=0.17, p=$ 0.01 ) but not in the AR-AD or healthy control group. A significant interaction between NPI and the groups confirmed that this effect was higher in those with preclinical AD than the other individuals $(\beta=0.44, \mathrm{SE}=0.19, p=0.02)$. Subcomponent analysis indicated that only the combined sleep/ nighttime behavior disorders and irritability/lability components of NPI predicted global $\left[{ }^{18} \mathrm{~F}\right] \mathrm{FDG}$ uptake decline in the preclinical AD group $(\beta=$ $0.51, \mathrm{SE}=0.17, p=0.008)$. NPI scores did not predict ADNI-mem scores in any of the groups at baseline or over 2 years.

The voxel-based analysis indicated that high NPI scores predicted regional $\left[{ }^{18} \mathrm{~F}\right] \mathrm{FDG}$ uptake decline over 2 years in the PCC and vmPFC of individuals with preclinical AD (figure 2). The mean $\left[{ }^{18} \mathrm{~F}\right] \mathrm{FDG}$ uptake

\begin{tabular}{|c|c|c|c|c|}
\hline & Healthy controls $(n=22$ ) & AR-AD $(n=60)$ & Preclinical AD $(n=33)$ & $p$ Value \\
\hline Age, mean (SD), y & $75.16(7.17)$ & $74.08(6.54)$ & $76.86(6.24)$ & 0.15 \\
\hline Male, n (\%) & $14(63.6)$ & $34(56.7)$ & $13(39.4)$ & 0.15 \\
\hline Education, mean (SD), y & $17.77(2.70)$ & $16.38(2.73)$ & $16.15(2.33)$ & 0.06 \\
\hline MMSE score, mean (SD) & 29.05 (1.49) & $29.17(1.12)$ & $28.91(0.94)$ & 0.58 \\
\hline APOE $\& 4$ carriers, $n(\%)$ & $1(4.5)$ & $16(26.7)$ & $12(36.4)$ & 0.02 \\
\hline Follow-up, mean (SD), y & $2.01(0.07)$ & $1.99(0.17)$ & $2.02(0.16)$ & 0.55 \\
\hline CSF p-tau $181 p$, mean (SD), pg/mL & $19.38(2.63)^{a, b}$ & $36.28(15.08)^{b, c}$ & $46.21(16.67)^{\mathrm{a}, \mathrm{c}}$ & $<0.001$ \\
\hline$\left[{ }^{18} \mathrm{~F}\right]$ florbetapir, mean (SD) SUVR & $1.06(0.03)^{b}$ & $1.08(0.08)^{b}$ & $1.30(0.09)^{a, c}$ & $<0.001$ \\
\hline
\end{tabular}
at the 2-year follow-up declined below normality in the PCC $(t=2.89, p=0.006)$ (figure 3B) but remained within a normal range, compared with biomarkernegative individuals, in the vmPFC (figure 3A).

Table Baseline demographics and sample characteristics

Abbreviations: $A D=$ Alzheimer disease; $A R-A D=$ asymptomatic at risk for Alzheimer disease; MMSE = Mini-Mental State Examination; $\mathrm{p}$-tau $\operatorname{ta1}_{1 \mathrm{p}}=$ phosphorylated tau; SUVR = standardized uptake value ratio.

$p$ values were assessed with analyses of variance for each variable except sex and APOE $\varepsilon 4$, for which a $\chi^{2}$ test was performed.

a Post hoc analysis provided significant group differences from AR-AD.

${ }^{b}$ Post hoc analysis provided significant group differences from preclinical AD.

${ }^{c}$ Post hoc analysis provided significant group differences from healthy controls. 
Figure 1 Individuals with preclinical AD with worse NPS have higher $\left[{ }^{18} \mathrm{~F}\right] \mathrm{FDG}$ uptake in PCC, vmPFC, Al at baseline

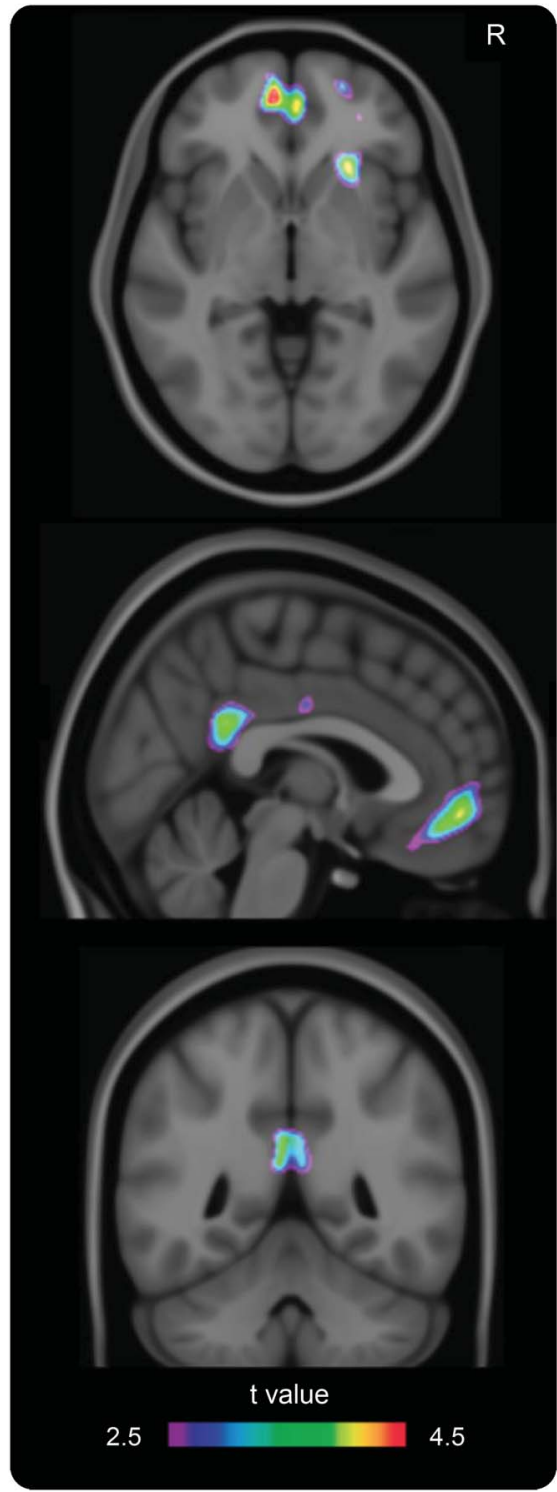

Statistical parametric map overlaid on a structural MRI scan shows regions in the PCC, vmPFC, and right Al where higher $\left[{ }^{18} \mathrm{~F}\right] F D G$ uptake was found in individuals with preclinical $A D$ with both amyloid and tau pathologies and higher NPI scores at baseline. The analysis was corrected for age, sex, education, and $A P O E \& 4$ status and multiple comparisons corrected with a false discovery rate corrected at $p<0.001 . A D=$ Alzheimer disease; $\mathrm{Al}=$ anterior insula; $\left[{ }^{18} \mathrm{~F}\right] \mathrm{FDG}=\left[{ }^{18} \mathrm{~F}\right]$ fluorodeoxyglucose; NPI = Neuropsychiatric Inventory; NPS = neuropsychiatric symptoms; $\mathrm{PCC}=$ posterior cingulate cortex; $\mathrm{VmPFC}=$ ventromedial prefrontal cortex.

DISCUSSION Our study showed that the magnitude of NPS is linked to a transient metabolic dysfunction in limbic networks that are vulnerable to early $\mathrm{AD}$ pathophysiology in individuals with preclinical AD. While individuals with preclinical $\mathrm{AD}$ with higher NPI scores had higher $\left[{ }^{18} \mathrm{~F}\right] \mathrm{FDG}$ uptake in the PCC, vmPFC, and right AI at baseline, high NPI
Figure 2 NPI predicts 2-year [ ${ }^{18}$ F]FDG uptake decline in PCC and vmPFC of individuals with preclinical $A D$

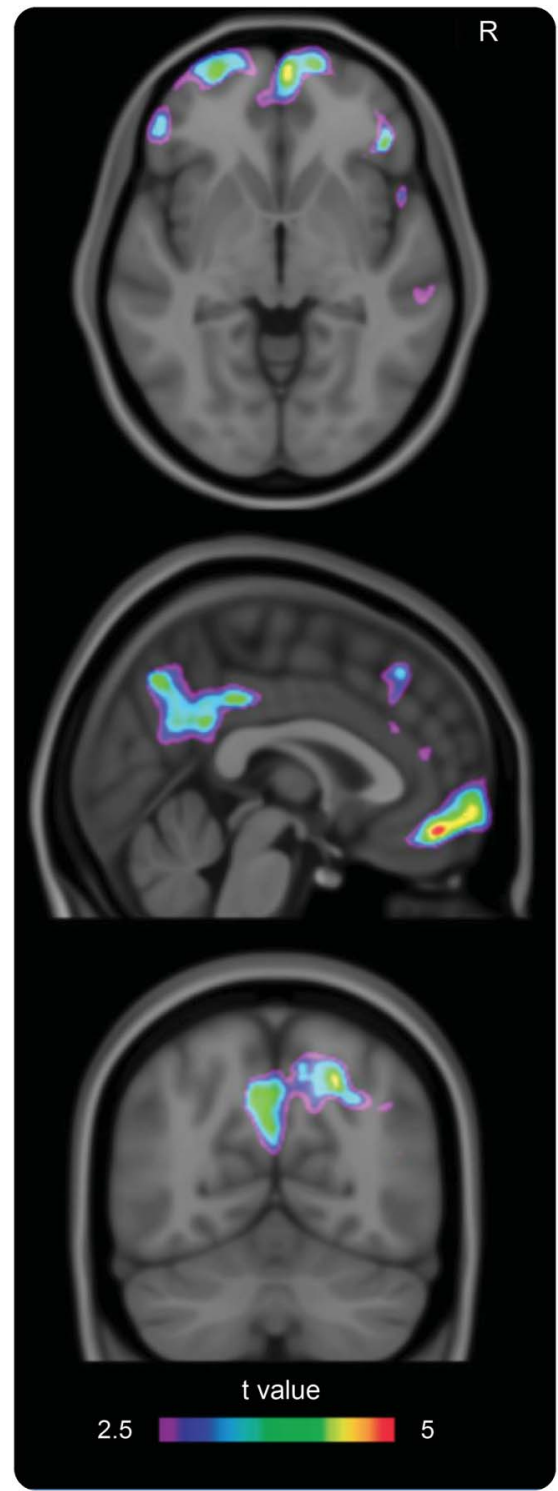

Statistical parametric map overlaid on a structural MRI scan showed regions in the PCC and vmPFC where 2-year $\left.{ }^{[18} \mathrm{F}\right] F D G$ uptake decline occurred as a function of baseline $\mathrm{NPI}$ in individuals with preclinical AD with both amyloid and tau pathologies. The analysis was corrected for age, sex, education, and APOE $\varepsilon 4$ status and multiple comparisons corrected with a false discovery rate corrected at $p<$ $0.001 . A D=$ Alzheimer disease; $\left[{ }^{18} \mathrm{~F}\right] F D G=\left[{ }^{18} \mathrm{~F}\right]$ fluorodeoxyglucose; $\mathrm{NPI}=$ Neuropsychiatric Inventory; $\mathrm{PCC}=$ posterior cingulate cortex; $\mathrm{vmPFC}=$ ventromedial prefrontal cortex.

scores predicted subsequent hypometabolism in the PCC. These metabolic dysfunctions were driven by the sleep/nighttime behavior disorders and irritability components of the NPI.

The finding of worse NPS with higher $\left[{ }^{18} \mathrm{~F}\right] \mathrm{FDG}$ uptake in the PCC, vmPFC, and right $\mathrm{AI}$ in individuals with preclinical $\mathrm{AD}$ at baseline suggests a link 
Figure 3 NPI predicts hypometabolism in PCC but not in vmPFC in individuals with preclinical $A D$

A

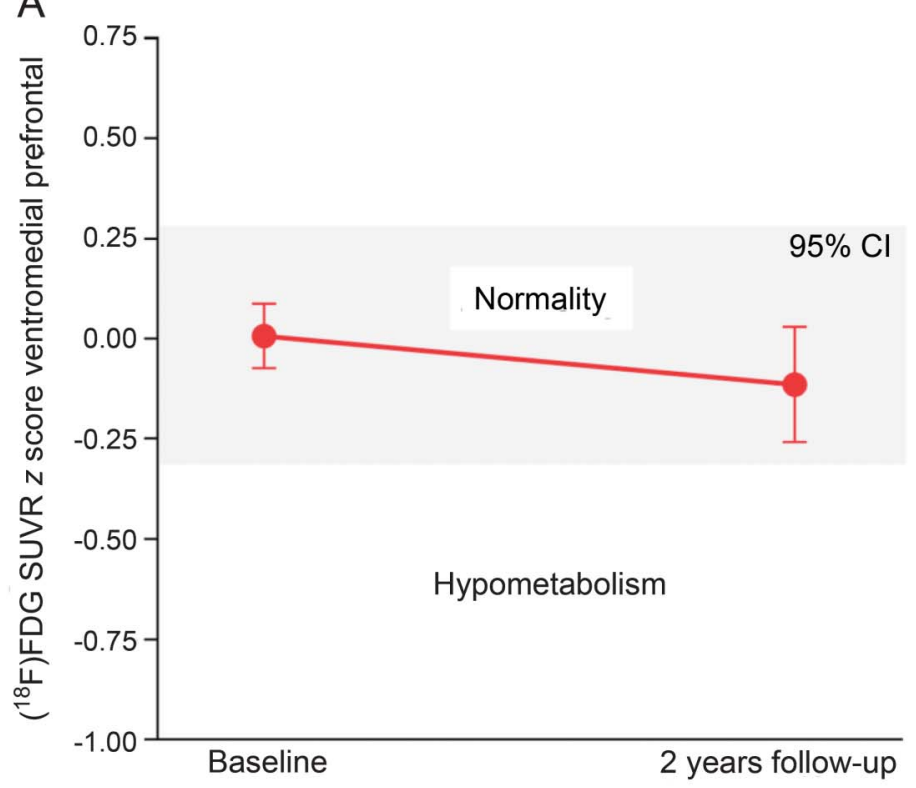

B

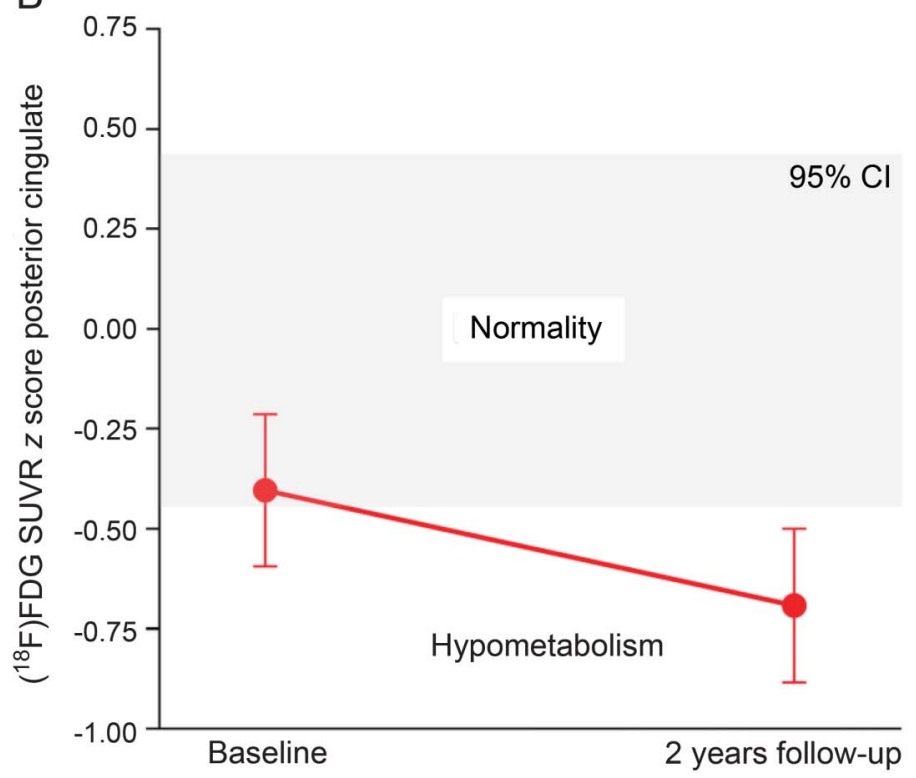

The dots represent the $z$-scored mean [ $\left.{ }^{18} F\right] F D G$ SUVR in (A) vmPFC and (B) PCC in individuals with preclinical $A D$ at baseline and the 2-year follow-up. The 2-year follow-up mean $\left[{ }^{18} \mathrm{~F}\right] \mathrm{FDG}$ uptake in PCC was lower $(95 \% \mathrm{Cl})$ than the mean of biomarker-negative individuals at follow-up, which suggests the presence of hypometabolism. However, the 2-year follow-up mean $\left[{ }^{18} F\right] F D G$ uptake in vmPFC remained within normal range in relation to biomarkernegative individuals. $A D=$ Alzheimer disease; $\mathrm{Cl}=$ confidence interval; $\left[{ }^{18} \mathrm{~F}\right] \mathrm{FDG}=\left[{ }^{18} \mathrm{~F}\right]$ fluorodeoxyglucose; NPI = Neuropsychiatric Inventory; $\mathrm{PCC}=$ posterior cingulate cortex; SUVR = standardized uptake value ratio; VMPFC $=$ ventromedial prefrontal cortex.

between specific brain regions involving early $\mathrm{AD}$ pathophysiology and behavioral control. Our results are in line with recent evidence from both functional and metabolic imaging studies. For example, a positive correlation has been shown between increased functional connectivity in the anterior cingulate and right insula regions of the salience network (SN) and irritability, agitation, disinhibition, aberrant motor behavior, and euphoria symptoms in patients with $\mathrm{AD},{ }^{19}$ while NPS changes have been proposed to reflect aberrant increases in SN functional connectivity. ${ }^{20}$ Most recently, NPS are found to be associated with increased glucose metabolism in the left insula, anterior cingulate gyrus, and superior frontal gyrus of patients with early-onset $\mathrm{AD} .^{5}$ Together, these results support a metabolic dysfunction in limbic networks affected by early AD pathophysiology, leading to NPS manifestations.

The AI, which is a key node of the $\mathrm{SN}$, plays a key role in generating appropriate behavioral responses by integrating affective, homeostatic, and higher-order cognitive processes. ${ }^{21}$ Hyperactivity in the AI may lead to enhanced salience detection, which is associated with NPS such as irritability or anxiety, ${ }^{19,22}$ while hypoactivity in the $\mathrm{AI}$ is associated with cognitive slowing and attentional deficits. ${ }^{23}$ The vmPFC regulates behavioral responses particularly in the context of changing reinforcement contingencies, emotional regulation, and decision-making tasks. ${ }^{24,25}$ Higher NPI scores and higher $\left[{ }^{18} \mathrm{~F}\right] \mathrm{FDG}$ uptake in the right $\mathrm{AI}$ and $\mathrm{vmPFC}$ at baseline may explain the enhanced neuropsychological presentations in these cognitively normal individuals who have both amyloid and tau pathologies.

Our findings of higher $\left[{ }^{18} \mathrm{~F}\right] \mathrm{FDG}$ uptake in the PCC in individuals with preclinical $\mathrm{AD}$ with higher NPI scores at baseline may represent a change in tissue metabolism in response to amyloid-related neurotoxicity to stabilize neuropsychiatric manifestations in the early stages of the disease. ${ }^{26}$ The PCC, a central part of the default mode network, is a highly connected and metabolically active region that plays a key role in supporting cognition. ${ }^{27}$ While hypometabolism in the PCC has been consistently observed in early $\mathrm{AD}$ pathophysiology, ${ }^{28}$ our findings suggest an early and transient neuronal compensation as a manifestation of cognitive reserve to preserve function that has been shown previously in cognitively normal individuals and those with $\mathrm{MCI}$ in association with amyloid deposition. ${ }^{26,29}$ As amyloid pathology progressively accumulates in the brain, metabolic decline occurs in response to the demands of the neuronal injury. ${ }^{26}$ In line with this concept, we showed that individuals with preclinical AD with worse NPS had subsequent hypometabolism in the PCC at the 2-year follow-up.

In addition, sleep/nighttime behavior disorders and irritability/lability were the NPI components that drove the metabolic dysfunctions in individuals with preclinical $\mathrm{AD}$. Both symptoms have been shown to be associated with early $\mathrm{AD}$ pathophysiology. For example, in the National Alzheimer's Coordinating Center data, noncognitive symptoms of early $\mathrm{AD}$ presented in 3 phases, and both irritability and 
nighttime behavior changes were found to be some of the first symptoms to occur. ${ }^{4}$ In a longitudinal study of the Massachusetts Alzheimer's Disease Research Center Cohort in which participants were cognitively normal, had subjective cognitive concerns, or were diagnosed with MCI, worse affective factor score predicted time to progression to a worse diagnosis in all 3 groups, driven by depression, agitation, and irritability. ${ }^{30}$ In the Mayo Clinic Study of Aging, baseline irritability in cognitively normal adults increased the risk of developing MCI over a median of 5 years (hazard ratio 1.84, 95\% confidence interval 1.312.58). ${ }^{31}$ Irritability and lability are NPS linked to abnormal emotional processing, associated with the vmPFC and PCC. ${ }^{25,32}$ In fact, reduced volume of the PCC gray matter has been shown to contribute to emotional instability. ${ }^{33}$ Sleep disturbances are common symptoms of AD and may be early indicators of amyloid pathology and dementia. ${ }^{34}$ Sleep deprivation can exacerbate amyloid pathology and mediate neuronal injury, while circadian dysfunction may also lead to cerebral oxidative stress and synaptic damage. ${ }^{34}$ Worse sleep quality is also shown to be associated with amyloid deposition in the preclinical AD stage. ${ }^{35}$ Patients with obstructive sleep apnea and daytime somnolence have decreased brain metabolism in the precuneus, middle and posterior cingulate gyrus, and parieto-occipital and prefrontal cortices, ${ }^{36}$ while healthy adults with dream enactment behavior have significantly lower glucose metabolism in brain regions, including the PCC. ${ }^{37}$ Hence, the finding of sleep/nighttime behavior disorders and irritability/ lability symptoms predicting hypometabolism in the PCC supports these NPS as early manifestations of AD pathophysiology.

Although baseline NPI score predicted $\left[{ }^{18} \mathrm{~F}\right] \mathrm{FDG}$ uptake decline, it did not predict memory decline using the ADNI-mem. Given that our study population is composed of a group of highly educated individuals, high cognitive reserve and education might play a role in stabilizing the cognitive function in the early stages of the disease. ${ }^{9,38,39}$ In addition, NPS may precede cognitive manifestations in early $\mathrm{AD}$ pathophysiology. In the Alzheimer's Disease Cooperative Study Prevention Instrument Project, the presence of baseline behavioral symptoms predicts conversion to a CDR score $\geq 0.5$ over 4 years in cognitively healthy older individuals, ${ }^{40}$ while in the National Alzheimer's Coordinating Center Data, a significantly earlier presence of NPS is found in cognitively normal people who progressed in CDR score compared to those who did not progress. ${ }^{4}$ This further supports NPS as early manifestations of AD pathophysiology.

There are limitations to our study. Although NPI is the most widely used instrument to measure NPS, it was initially designed for dementia studies, not for preclinical AD studies. ${ }^{11}$ Hence, the sensitivity of NPI in diagnosing NPS in individuals with preclinical $\mathrm{AD}$ is not known. In addition, given that the NPI is based on responses from an informed caregiver, the NPI scores may not accurately reflect the NPS of study participants. The ADNI database is made up of highly educated individuals who volunteered to participate in the study that focused on $\mathrm{AD}$ research. This may introduce selection bias in that the study population is a self-selected group of individuals who may have concerns about their cognition. The selfselected character of our study population and the relatively small sample size of our study limit the generalizability of our findings to a broader community. Therefore, our findings will need to be confirmed in a larger population-based cohort.

Our findings support an emerging conceptual framework that NPS, driven by sleep behavior and irritability domains, are early manifestations of $\mathrm{AD}$ pathophysiology. Therefore, early NPS may further contribute to the characterization of the preclinical $\mathrm{AD}$ stage.

\section{AUTHOR CONTRIBUTIONS}

Kok Pin Ng: study concept, design, analysis and interpretation of data, compose figures and manuscript draft. Tharick A. Pascoal: study design, analysis and interpretation of data, compose figures and manuscript draft. Sulantha Mathotaarachchi, Chang-Oh Chung, Andréa L. Benedet, Monica Shin, Min Su Kang: image data processing and manuscript draft. Xiaofeng Li, Maowen Ba: manuscript draft. Nagaendran Kandiah: study concept, design and manuscript draft. Pedro Rosa-Neto, Serge Gauthier: study concept, design, study supervision, and critical review of manuscript for intellectual content.

\section{STUDY FUNDING}

This work was supported by the Canadian Institutes of Health Research (CIHR) [MOP-11-51-31], Canadian Consortium of Neurodegeneration in Aging (CIHR-CCNA), the Weston Brain Institute, and the Alzheimer's Association (NIRG-08-92090). Pedro Rosa-Neto is supported by the Fonds de la recherche en santé du Québec (chercheur boursier), and Kok Pin Ng is supported by the National Medical Research Council, Research Training Fellowship, Singapore. Pedro Rosa-Neto and Serge Gauthier are CIHR-CCNA members. Data collection and sharing for this project were funded by the ADNI (NIH grant U01 AG024904) and Department of Defense ADNI (award W81XWH-12-2-0012). ADNI is funded by the National Institute on Aging, by the National Institute of Biomedical Imaging and Bioengineering, and through generous contributions from the following: AbbVie, Alzheimer's Association; Alzheimer's Drug Discovery Foundation; Araclon Biotech; BioClinica, Inc; Biogen; Bristol-Myers Squibb Co; CereSpir, Inc; Cogstate; Eisai Inc; Elan Pharmaceuticals, Inc; Eli Lilly and Co; EuroImmun; F. Hoffmann-La Roche Ltd and its affiliated company Genentech, Inc; Fujirebio; GE Healthcare; IXICO Ltd; Janssen Alzheimer Immunotherapy Research \& Development, LLC; Johnson \& Johnson Pharmaceutical Research \& Development LLC; Lumosity; Lundbeck; Merck \& Co, Inc; Meso Scale Diagnostics, LLC; NeuroRx Research; Neurotrack Technologies; Novartis Pharmaceuticals Corp; Pfizer Inc; Piramal Imaging; Servier; Takeda Pharmaceutical Co; and Transition Therapeutics. The CIHR is providing funds to support ADNI clinical sites in Canada. Private sector contributions are facilitated by the Foundation for the NIH (www.fnih.org). The grantee organization is the Northern California Institute for Research and Education, and the study is coordinated by the Alzheimer's Therapeutic Research Institute at the University of 
Southern California. ADNI data are disseminated by the Laboratory for Neuro Imaging at the University of Southern California.

\section{DISCLOSURE}

K. Ng, T. Pascoal, S. Mathotaarachchi, C. Chung, A. Benedet, M. Shin M. Kang, X. Li, M. Ba, N. Kandiah, and P. Rosa-Neto report no disclosures relevant to the manuscript. S. Gauthier received honoraria for serving on the scientific advisory boards of Alzheon, Axovant, Lilly, Lundbeck, Novartis, Schwabe, and TauRx and on the Data Safety Monitoring Board of a study sponsored by Eisai and studies run by the Alz heimer's Disease Cooperative Study and by the Alzheimer's Therapeutic Research Institute. Research was funded by CIHR and NIH. Go to Neurology.org for full disclosures.

Received October 14, 2016. Accepted in final form February 17, 2017.

\section{REFERENCES}

1. Lyketsos CG, Carrillo MC, Ryan JM, et al. Neuropsychiatric symptoms in Alzheimer's disease. Alzheimers Dement 2012;7:532-539

2. Fischer CE, Ismail Z, Schweizer TA. Delusions increase functional impairment in Alzheimer's disease. Dement Geriatr Cogn Disord 2012;33:393-399.

3. Teng E, Lu PH, Cummings JL. Neuropsychiatric symptoms are associated with progression from mild cognitive impairment to Alzheimer's disease. Dement Geriatr Cogn Disord 2007;24:253-259.

4. Masters MC, Morris JC, Roe CM. "Noncognitive” symptoms of early Alzheimer disease : a longitudinal analysis. Neurology 2015;84:617-622.

5. Ballarini T, Iaccarino L, Magnani G, et al. Neuropsychiatric subsyndromes and brain metabolic network dysfunctions in early onset Alzheimer's disease. Hum Brain Mapp 2016;37:4234-4247.

6. Ismail Z, Smith EE, Geda Y, et al. Neuropsychiatric symptoms as early manifestations of emergent dementia: provisional diagnostic criteria for mild behavioral impairment. Alzheimers Dement 2016;12:195-202.

7. Ruider H, Krell-Roesch J, Stokin G, et al. FDG-PET of the brain and neuropsychiatric symptoms in normal cognitive aging: the Mayo Clinic Study of Aging. J Alzheimers Dis 2016;53:1609-1616.

8. Mosconi L, Berti V, Glodzik L, Pupi A, De Santi S, De Leon MJ. Pre-clinical detection of Alzheimer's disease using FDG-PET, with or without amyloid imaging. J Alzheimers Dis 2010;20:843-854.

9. Dubois B, Hampel H, Feldman $\mathrm{HH}$, et al. Preclinical Alzheimer's disease: definition, natural history, and diagnostic criteria. Alzheimers Dement 2016;12:292323.

10. Edmonds EC, Delano-Wood L, Galasko DR, Salmon DP, Bondi MW; Alzheimer's Disease Neuroimaging Initiative. Subtle cognitive decline and biomarker staging in preclinical Alzheimer's disease. J Alzheimers Dis 2015; 47:231-242.

11. Cummings JL, Mega M, Gray K, Rosenberg-Thompson S, Carusi DA, Gornbein J. The Neuropsychiatric Inventory: comprehensive assessment of psychopathology in dementia. Neurology 1994;44:2308-2314.

12. Crane PK, Carle A, Gibbons LE, et al. Development and assessment of a composite score for memory in the Alzheimer's Disease Neuroimaging Initiative (ADNI). Brain Imaging Behav 2012;6:502-516.

13. Shaw LM, Vanderstichele H, Knapik-Czajka M, et al. Cerebrospinal fluid biomarker signature in Alzheimer's
Disease Neuroimaging Initiative subjects. Ann Neurol 2009;65:403-413.

14. Zijdenbos AP, Forghani R, Evans AC. Automatic "pipeline" analysis of 3-D MRI data for clinical trials: application to multiple sclerosis. IEEE Trans Med Imaging 2002; 21:1280-1291.

15. Pascoal TA, Mathotaarachchi S, Mohades S, et al. Amyloid- $\beta$ and hyperphosphorylated tau synergy drives metabolic decline in preclinical Alzheimer's disease. Mol Psychiatry 2017;22:306-311.

16. Fawcett T. An introduction to ROC analysis. Pattern Recognit Lett 2006;27:861-874.

17. Jack CR, Lowe VJ, Weigand SD, et al. Serial PIB and MRI in normal, mild cognitive impairment and Alzheimer's disease: implications for sequence of pathological events in Alzheimer's disease. Brain 2009;132: 1355-1365.

18. R Development Core Team. R: a language and environment for statistical computing. R Found Stat Comput 2015;1:409.

19. Balthazar MLF, Pereira FRS, Lopes TM, et al. Neuropsychiatric symptoms in Alzheimer's disease are related to functional connectivity alterations in the salience network. Hum Brain Mapp 2014;35:1237-1246.

20. Zhou J, Seeley WW. Network dysfunction in Alzheimer's disease and frontotemporal dementia: implications for psychiatry. Biol Psychiatry 2014;75:565-573.

21. Menon V, Uddin LQ. Saliency, switching, attention and control: a network model of insula function. Brain Struct Funct 2010;214:655-667.

22. Stein MB, Simmons AN, Feinstein JS, Paulus MP. Increased amygdala and insula activation during emotion processing in anxiety-prone subjects. Am J Psychiatry 2007; 164:318-327.

23. Blanc F, Colloby SJ, Cretin B, et al. Grey matter atrophy in prodromal stage of dementia with Lewy bodies and Alzheimer's disease. Alzheimers Res Ther 2016;8: 31.

24. Zald DH, Andreotti C. Neuropsychological assessment of the orbital and ventromedial prefrontal cortex. Neuropsychologia 2010;48:3377-3391.

25. Etkin A, Büchel C, Gross JJ. The neural bases of emotion regulation. Nat Rev Neurosci 2015;16:693-700.

26. Ashraf A, Fan Z, Brooks DJ, Edison P. Cortical hypermetabolism in MCI subjects: a compensatory mechanism? Eur J Nucl Med Mol Imaging 2014;42:447-458.

27. Leech R, Sharp DJ. The role of the posterior cingulate cortex in cognition and disease. Brain 2014;137:12-32.

28. Johnson Ka, Jones K, Holman BL, et al. Preclinical prediction of Alzheimer's disease using SPECT. Neurology 1998;50:1563-1571.

29. Ossenkoppele R, Madison C, Oh H, Wirth M, Van Berckel BNM, Jagust WJ. Is verbal episodic memory in elderly with amyloid deposits preserved through altered neuronal function? Cereb Cortex 2014;24: 2210-2218.

30. Donovan NJ, Amariglio RE, Zoller AS, et al. Subjective cognitive concerns and neuropsychiatric predictors of progression to the early clinical stages of Alzheimer disease. Am J Geriatr Psychiatry 2014;22:1642-1651.

31. Geda YE, Roberts RO, Mielke MM, et al. Baseline neuropsychiatric symptoms and the risk of incident mild cognitive impairment: a population-based study. Am J Psychiatry 2014;171:572-581. 
32. Phillips ML, Drevets WC, Rauch SL, Lane R. Neurobiology of emotion perception II: implications for major psychiatric disorders. Biol Psychiatry 2003;54:515-528.

33. Hazlett EA, New AS, Newmark R, et al. Reduced anterior and posterior cingulate gray matter in borderline personality disorder. Biol Psychiatry 2005;58:614-623.

34. Musiek ES, Xiong DD, Holtzman DM. Sleep, circadian rhythms, and the pathogenesis of Alzheimer disease. Exp Mol Med 2015;47:e148.

35. Ju YES, McLeland JS, Toedebusch CD, et al. Sleep quality and preclinical Alzheimer disease. JAMA Neurol 2013;70: 587-593.

36. Yaouhi K, Bertran F, Clochon P, et al. A combined neuropsychological and brain imaging study of obstructive sleep apnea. J Sleep Res 2009;18:36-48.
37. Caselli RJ, Chen K, Bandy D, et al. A preliminary fluorodeoxyglucose positron emission tomography study in healthy adults reporting dream-enactment behavior. Sleep 2006;29:927-933.

38. Roe CM, Xiong C, Miller JP, Morris JC. Education and Alzheimer disease without dementia: support for the cognitive reserve hypothesis. Neurology 2007;68:223228.

39. Stern Y. Cognitive reserve and Alzheimer disease. Alzheimer Dis Assoc Disord 2006;20:S69-S74.

40. Banks SJ, Raman R, He F, et al. The Alzheimer's Disease Cooperative Study Prevention Instrument Project: longitudinal outcome of behavioral measures as predictors of cognitive decline. Dement Geriatr Cogn Dis Extra 2014;4: 509-516.

\section{This Week's Neurology ${ }^{\circledR}$ Podcast}

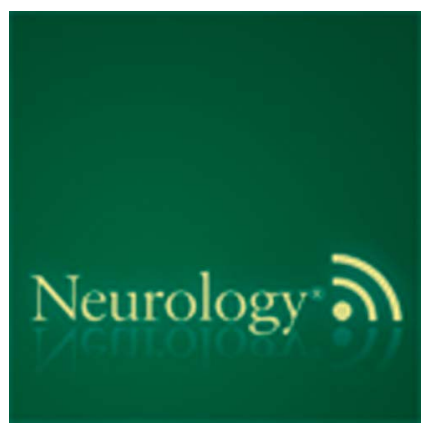

Serum neurofilament light as a biomarker for mild traumatic brain injury in contact sports (see p. 1788)

This podcast begins and closes with Dr. Robert Gross, Editor-inChief, briefly discussing highlighted articles from the May 9, 2017, issue of Neurology. In the first segment, Dr. Kristen Heinan interviews Dr. Pashtun Shahim about his paper on serum neurofilament light as a biomarker for mild traumatic brain injury. In the next segment, Dr. Alberto Espay and Dr. David Standaert discuss a recent debate regarding the continuation of adhering to biomarker validation efforts on clinical criteria for our "What's Trending" feature of the week.

Disclosures can be found at Neurology.org.

At Neurology.org, click on "RSS" in the Neurology Podcast box to listen to the most recent podcast and subscribe to the RSS feed.

CME Opportunity: Listen to this week's Neurology Podcast and earn 0.5 AMA PRA Category

1 CME Credits ${ }^{\mathrm{TM}}$ by answering the multiple-choice questions in the online Podcast quiz.

\section{Call for Applications for NeuroLearn ${ }^{\mathrm{SM}}$ Editor-in-Chief}

Would you like to lead and advise on program strategy, innovation, and content for the AAN's suite of exclusive online education courses designed to address relevant clinical and practice topics? For a detailed position description, desired qualifications, and to apply, visit AAN.com/view/ NeuroLearn. Application deadline: May 30. 


\section{Neurology}

Neuropsychiatric symptoms predict hypometabolism in preclinical Alzheimer disease

Kok Pin Ng, Tharick A. Pascoal, Sulantha Mathotaarachchi, et al.

Neurology 2017;88;1814-1821 Published Online before print April 12, 2017

DOI 10.1212/WNL.0000000000003916

This information is current as of April 12, 2017

Updated Information \&
Services
Supplementary Material

References

Citations

Subspecialty Collections

Permissions \& Licensing

Reprints including high resolution figures, can be found at:

http://n.neurology.org/content/88/19/1814.full

Supplementary material can be found at:

http://n.neurology.org/content/suppl/2017/04/12/WNL.0000000000003 916.DC1

This article cites 40 articles, 4 of which you can access for free at: http://n.neurology.org/content/88/19/1814.full\#ref-list-1

This article has been cited by 6 HighWire-hosted articles: http://n.neurology.org/content/88/19/1814.full\#\#otherarticles

This article, along with others on similar topics, appears in the following collection(s):

Alzheimer's disease

http://n.neurology.org/cgi/collection/alzheimers_disease

Cognitive neuropsychology in dementia

http://n.neurology.org/cgi/collection/cognitive_neuropsychology_in_de mentia

PET

http://n.neurology.org/cgi/collection/pet

Information about reproducing this article in parts (figures,tables) or in its entirety can be found online at:

http://www.neurology.org/about/about_the_journal\#permissions

Information about ordering reprints can be found online:

http://n.neurology.org/subscribers/advertise

Neurology ${ }^{\circledR}$ is the official journal of the American Academy of Neurology. Published continuously since 1951, it is now a weekly with 48 issues per year. Copyright Copyright @ 2017 The Author(s). Published by Wolters Kluwer Health, Inc. on behalf of the American Academy of Neurology. All rights reserved. Print ISSN: 0028-3878. Online ISSN: 1526-632X.

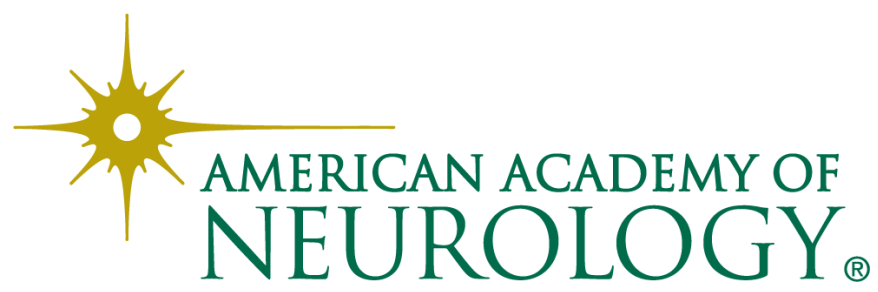

